\title{
The Macroeconomics of TANSTAAFL
}

\author{
Volker Grossmann, ${ }^{\dagger}$ Thomas M. Steger, ${ }^{\ddagger}$ and Timo Trimborn ${ }^{\S}$
}

November 21, 2011

\begin{abstract}
This paper shows that dynamic inefficiency can occur in dynamic general equilibrium models with fully optimizing, infinitely-lived households even in a situation with underinvestment. We identify necessary conditions for such a possibility and illustrate it in a standard R\&D-based growth model. Calibrating the model to the US, we show that a moderate increase in the R\&D subsidy indeed leads to an intertemporal free lunch (i.e., an increase in per capita consumption at all times). Hence, Milton Friedman's conjecture There ain't no such thing as a free lunch (TANSTAAFL) may not apply.
\end{abstract}

Key words: Intertemporal free lunch; Dynamic inefficiency; R\&D-based growth; Transitional dynamics.

JEL classification: E20, H20, O41.

*Acknowledgements: We are grateful to participants at the workshop on "Innovation" at the 41th "Ottobeuren-Seminar" for helpful comments and suggestions as well as to numerous colleagues which whom we discussed our research agenda on optimal dynamic growth policy over the last years.

${ }^{\dagger}$ University of Fribourg; CESifo, Munich; Institute for the Study of Labor (IZA), Bonn. Address: University of Fribourg, Department of Economics, Bd. de Pérolles 90, 1700 Fribourg, Switzerland. E-mail: volker.grossmann@unifr.ch.

${ }^{\ddagger}$ University of Leipzig; CESifo, Munich. Address: Institute for Theoretical Economics, Grimmaische Strasse 12, 04109 Leipzig, Germany, Email: steger@wifa.uni-leipzig.de.

$\S$ University of Hannover. Address: Institute for Macroeconomics, Königsworther Platz 1, 30167 Hannover, Germany, Email: trimborn@vwl.uni-hannover.de. 


\section{Introduction}

The saying There ain't no such thing as a free lunch, abbreviated by the acronym TANSTAAFL and popularized in economics by Milton Friedman (1975), expresses the insight that every benefit comes at a cost. There is one general exception to this rule. If resources are being used inefficiently, it is possible to get "something for nothing".

In dynamic macroeconomics, such a possibility is referred to as dynamic inefficiency. That is, one may ask if it is possible to implement an allocation of resources such that per capita consumption increases for some periods and does not decline for any period. As is well-known, the Solow model exhibits dynamic inefficiency if the saving rate lies above its golden rule level such that capital is overaccumulated (Phelps, 1966). However, this example is based on a model without optimizing behavior on the side of households. In an overlapping-generations context, dynamic inefficiency may result since current generations do not take changes in the future interest rate into account when deciding on their saving rate (e.g. Abel et al., 1989).

This paper shows that dynamic inefficiency can also occur in an optimizing agent framework with infinitely lived households and underinvestment. That is, we start out with a situation where, from a long run perspective, resources allocated to the accumulation of (at least) one accumulable production factor are too low in market equilibrium compared to the social optimum. Note that, for instance, in the Solowmodel in such a situation the economy is always dynamically efficient.

The contribution of our paper is twofold. First, we identify necessary conditions for the possibility of dynamic inefficiency in a situation with underinvestment within the widely-used class of dynamic general equilibrium models with one consumption good and fully optimizing, infinitely-lived agents. Second, we employ a calibrated version of the standard (semi-)endogenous growth model of Jones (1995) to show that the US economy may indeed be dynamically inefficient. That is, stimulating R\&D investment by raising the $R \& D$ subsidy not only raises intertemporal welfare but, at the first glance surprisingly, also enables an increase in per capita consumption at all times compared to the baseline scenario of no policy change. The reason is that 
households immediately increase their consumption rate due to their expectation of future productivity advances after the $\mathrm{R} \& \mathrm{D}$ underinvestment problem is tackled. Thus, the accumulation of physical capital slows down in the initial transition phase to the new steady state while more resources are devoted to knowledge accumulation. The decrease in the rate of investment in physical capital then enables an intertemporal free lunch. Hence, our analysis suggests that TANSTAAFL may not even apply in advanced economies, due to likely $R \& D$ underinvestment. In our calibrated economy, only for large increases in the subsidy rate, possibly beyond the socially optimal long run rate, per capita consumption drops initially.

More generally, we identify the following necessary conditions for an intertemporal free lunch: (i) there is a policy intervention which affects an allocation variable governing the evolution of the distorted accumulable factor; (ii) the number of allocation variables exceeds the number of resource constraints by at least two, i.e., there are at least two degrees of freedom. Within the Jones (1995) model, for instance, an increase in the R\&D subsidy induces a reallocation of labor towards the $R \& D$ sector. This requires a first allocation variable to be set freely. For an intertemporal free lunch to be feasible, i.e. for consumption not to decrease initially, capital accumulation has to decelerate, which requires a second degree of freedom. This response to the R\&D policy intervention allows for consumption smoothing in the presence of a substantial positive wealth effect.

The paper is organized as follows. Section 2 lies out a general, dynamic macroeconomic framework with one consumption good and identifies necessary conditions for dynamic inefficiency. Section 3 calibrates a R\&D-based growth model which fulfills these conditions and shows that the economy is indeed dynamically inefficient. Section 4 briefly discusses the possibility of an intertemporal free lunch in various other widely-used endogenous growth models. The last section concludes. 


\section{On the Mechanics of an Intertemporal Free Lunch}

This section discusses the necessary conditions for an intertemporal free lunch. To be precise we start with a definition of an intertemporal free lunch.

Definition 1. Let $c_{B}(t)$ denote the time path of per capita consumption in the baseline scenario (i.e. without any policy change) and let $c_{A}(t)$ denote the time path of per capita consumption in the alternative scenario (i.e. after a policy change). An intertemporal free lunch is possible (i.e. an economy is dynamically inefficient) if and only if there is a feasible policy measure such that per capita consumption in the alternative scenario $c_{A}(t)$ increases relative to per capita consumption in the baseline scenario $c_{B}(t)$ for some $t \geq 0$ and does not decline for any $t \geq 0$, i.e. $c_{A}(t)>c_{B}(t)$ for at least some $t$ and $c_{A}(t) \geq c_{B}(t)$ for all $t$.

Notice that we require that an intertemporal free lunch can be realized by a feasible policy intervention in a market equilibrium.

As it may be trivial to discuss the case where dynamic inefficiency occurs in the case of overaccumulation, we focus on the case where, compared to the social optimum, the market equilibrium involves underinvestment in (at least) one accumulable production factor. The economic logic behind an intertemporal free lunch in this case may be sketched as follows. Consider a policy intervention which changes the allocation of resources in the market equilibrium such that the hitherto underaccumulated input factor gets accumulated in an accelerated fashion. Ceteris paribus, this reduces per capita consumption at least initially. However, an intertemporal free lunch requires that the production of consumption goods remains at least constant initially. Thus, in addition to the allocation variable which causes accelerated accumulation of the distorted factor, there has to be a second allocation variable which can adjust freely in response to the policy intervention. An adjustment of this second allocation variable could ensure that the initial consumption level does not decrease. The requirement excludes cases where the accumulation of one input factor is a by-product of the accumulation of another input factor, in the sense that both are driven by the same allocation variable. An 
example for such a case is the Arrow-Romer model (Romer, 1986), discussed in section 4 .

To illustrate, consider a dynamic, closed economy framework with a single consumption good, chosen as numeraire. For simplicity, suppose there exists a representative household, who is fully rational, perfectly informed and acts as living infinitely. The size of a household, $N$, grows at constant exponential rate $n \geq 0$. His/her preferences are represented by the standard intertemporal utility function

$$
U=\int_{0}^{\infty} u(c(t)) e^{-(\rho-n) t} d t
$$

$\rho>0$, where $c(t)$ is per capita consumption at time $t \in \mathbb{R}_{+}$and $u$ denotes instantaneous utility. The size of the representative household equals labor supply to a perfect labor market (i.e., total employment is equal to $N$ ).

The production function $F$ of the homogenous final good is ${ }^{1}$

$$
Y=F\left(K_{1}^{Y}, K_{2}^{Y} \ldots, K_{I}^{Y}, L^{Y}\right)
$$

where $K_{i}^{Y}$ is the input of capital good $i \in\{1, \ldots, I\}$ and $L^{Y}$ is labor input into final production. Capital goods are factors which are accumulable by investments, whereas labor is non-accumulable (but may grow exogenously). ${ }^{2}$ From a social point of view, the $Y$-production technology may exhibit increasing returns to scale. The representative final goods producer privately perceives constant returns to scale. We distinguish between two classes of dynamic macroeconomic models.

One-sector models. Total supply of accumulable factor $i$ is denoted by $K_{i}$. Given initial level $K_{i}(0)$, it evolves according to ${ }^{3}$

$$
\dot{K}_{i}=G_{i}\left(Y^{i}\right)-\delta_{i} K_{i}
$$

\footnotetext{
${ }^{1}$ The time index is omitted whenever this does not lead to confusion.

${ }^{2}$ For simplicity, we consider one non-accumulable factor only. Generalization to more than one non-accumulable factor is straightforward.

${ }^{3} \dot{Z}$ denotes the derivative of a variable $Z$ with respect to time.
} 
where function $G_{i}(\cdot)$ gives us the gross increase of factor $i \in\{1, \ldots, I\}, Y^{i}$ denotes the amount of final output devoted to accumulation of factor $i$ and $\delta_{i} \geq 0$ the depreciation rate of capital good $i$. The $G_{i}(\cdot)$ are increasing functions.

As an example, consider the Solow-model. In this model, gross investment in physical capital (say, capital good 1) reads $G_{1}(\cdot)=Y^{1}=\mathfrak{s} Y$, where $\mathfrak{s}>0$ is the exogenous rate of investment out of the final good. ${ }^{4}$ It is well-known that the economy is dynamically inefficient if $\mathfrak{s}$ is above its "golden rule" level, which maximizes per capita consumption in steady state. By contrast, our goal is to examine the possibility of dynamic inefficiency in general equilibrium, infinite-horizon models with optimizing agents and underinvestment in the sense that, from a long run perspective, resources allocated to the accumulation of (at least) one accumulable production factor are too low from a social planer's point of view.

The economy's goods market clearing condition reads

$$
N c+\sum_{i=1}^{I} Y^{i}=Y
$$

Moreover, $L^{Y}=N$ and $K_{i}^{Y}=K_{i}$ for all $i$ (full employment conditions).

Per capita consumption $(c)$ as well as the arguments of functions $F$ and $G_{i}$ are called "allocation variables". Let $V$ denote the number of allocation variables and let $R$ be the number of constraints (restrictions). For the generic one-sector model the number of allocation variables $\left(c, K_{1}^{Y}, \ldots, K_{I}^{Y}, L^{Y}, Y^{1}, \ldots, Y^{I}\right)$ is $V=2 I+2$, whereas the number of constraints is $R=I+2$ (full employment conditions and (4)). We now argue that an intertemporal free lunch requires that $V-R=I \geq 2$. That is, there are at least two degrees of freedom in the allocation variables. In the one-sector model, this is equivalent to the requirement of at least two accumulable factors.

Let $q:=N c / Y$ denote the economy's consumption rate and denote by $\mathfrak{s}^{i}:=Y^{i} / Y$ the fraction of final output devoted to the accumulation of capital good $i$. We label $\mathfrak{s}^{i}$

\footnotetext{
${ }^{4}$ Mankiw, Romer and Weil (1992) present an augmented Solow-model with human capital as second accumulable factor, which evolves analogously to physical capital.
} 
the "investment rate of factor $i$ ". According to (4), we can write

$$
q=1-\sum_{i=1}^{I} \mathfrak{s}^{i}
$$

Obviously, if $I=1$, raising the only investment rate inevitably lowers the consumption rate at least initially. Thus, an economy with underinvestment is always dynamically efficient. However, if $I \geq 2$, it could be the case that by raising, say, investment rate $\mathfrak{s}^{1}$, forward-looking agents reduce, say, investment rate $\mathfrak{s}^{2}$ such that the consumption rate $q$ instantaneously rises despite increased accumulation of factor 1 .

Multi-sector models. In multi-sector models, the number of accumulable factors does not necessarily coincide with the degrees of freedom in the allocation variables. Denote total supply of accumulable factor $i$ by $K_{i}$. Given initial level $K_{i}(0)$, it evolves according to

$$
\dot{K}_{i}=G_{i}\left(K_{1}^{i}, K_{2}^{i} \ldots, K_{I}^{i}, L^{i}\right)-\delta_{i} K_{i}
$$

where function $G_{i}(\cdot)$ gives us the gross increase of factor $i \in\{1, \ldots, I\}, K_{j}^{i}$ is the amount of capital good $j \in\{1, \ldots, I\}$ devoted to the accumulation of capital good $i$, and $L^{i}$ is the amount of labor devoted to accumulation of factor $i$. Again, $\delta_{i} \geq 0$ is the depreciation rate of capital good $i$. We refer to arguments of the functions $G_{i}(\cdot)$ as allocation variables which govern the evolution of an accumulable factor. They are a subset of all allocation variables (recall that the total set also consists of per capita consumption and the arguments of production function $F$ ). The $G_{i}(\cdot)$ are increasing in the allocation variables.

The resource constraints read

$$
\begin{gathered}
L^{Y}+\sum_{i=1}^{I} L^{i}=N \\
K_{j}^{Y}+\sum_{i=1}^{I} K_{j}^{i}=K_{j} \text { for all } j \in\{1, \ldots, I\}
\end{gathered}
$$

Moreover, $N c=Y$. 
The number of allocation variables $\left(c, K_{1}^{Y}, \ldots, K_{I}^{Y}, L^{Y}, L^{1}, . ., L^{I}, K_{1}^{1}, \ldots, K_{I}^{I}\right)$ is $V=$ $2 I+2+I^{2}$, while the number of constraints, (7), (8) and $N c=Y$, reads $R=I+2$. There are at least two degrees of freedom in the allocation variables provided that $V-R=I(1+I) \geq 2$. This is already the case if $I \geq 1$. However, in a multisector model with just one accumulable input factor $(I=1)$, it is difficult to conceive where an intertemporal free lunch is possible. Both capital and labor input in final goods production have to be reallocated in a way (one increased, the other decreased) such that the accumulation of a sole capital good is accelerated and the production of consumption is held at least constant initially.

In the special case where $K_{j}^{i}=0$ for all $i, j \in\{1, \ldots, I\}$ and labor is used everywhere, we have $V=2 I+2$ and thus $V-R=I$. Thus, two degrees of freedom means that there are two accumulable factors, like in the one-sector model. If $L^{i}=0$ for all $i$ and all capital goods are used everywhere, then $V=I+2+I^{2}$ and thus $V-R=I^{2}$. Again, $V-R \geq 2$ requires $I \geq 2$.

Compound models. There may be a mix of both classes of models. In fact, the Jones (1995) model, discussed in section 3, belongs to this category. Consider a model with $I^{A}+I^{B}=I$ accumulable factors, where $I^{A} \geq 1$ factors are accumulated according to (3) and $I^{B} \geq 1$ are accumulated according to (6). If both labor and all capital goods enter function $F$ as well as functions $G_{i}$, there are $V-R=I^{A}+I^{B}\left(1+I^{B}\right)$ degrees of freedom. In the special case where $K_{j}^{i}=0$ for all $i, j$ and labor enters everywhere, we have $V-R=I^{A}+I^{B}$. If $L^{i}=0$ for all $i$ and all capital goods are used everywhere, then $V=I+2+I^{2}$ and thus $V-R=I^{A}+\left(I^{B}\right)^{2}$. In all cases, $I^{A} \geq 1$ and $I^{B} \geq 1$, which holds by assumption, implies $V-R \geq 2$. We conclude that an intertemporal free lunch cannot be a priori ruled out in compound models.

The discussion is summarized by the following proposition.

Proposition 1. Consider a closed economy with exogenous labor supply and one consumption good. Suppose that at least one accumulable factor is underaccumulated from a social point of view. An intertemporal free lunch is possible if the following necessary conditions simultaneously hold: (i) there is a policy intervention which affects 
an allocation variable governing the evolution of the distorted accumulable factor, (ii) there are at least two degrees of freedom in the allocation variables.

\section{Dynamic Inefficiency and R\&D-based Growth}

This section analyzes the possibility of an intertemporal free lunch in a widely-used R\&D-based growth model with both accumulation of knowledge and physical capital goods.

\subsection{The Jones (1995) Model}

Consider the R\&D-based growth model of Jones (1995). The final output technology is

$$
Y=\left(L^{Y}\right)^{1-\alpha} \sum_{i=1}^{A}\left(x_{i}\right)^{\alpha} d i
$$

with $0<\alpha<1$, where $L^{Y}$ denotes labor employed in final output production and $x_{i}$ the quantity of (physical) capital good $i \in\{1, \ldots, A\}$. (Time index $t$ is omitted.) One unit of final output can be transformed into one unit of each capital good and all capital goods depreciate at the same constant rate $\delta$. Thus, we can write the equations of motion for physical capital goods $\mathrm{as}^{5}$

$$
\dot{x}_{i}=Y^{i}-\delta x_{i}, i \in\{1, \ldots, A\} .
$$

The number of intermediate goods $A$ ("stock of knowledge") is an additional accumulable factor. (The total number of accumulable factors is $I=A+1$.) It expands through horizontal innovations according to

$$
\dot{A}=\tilde{\nu} A^{\phi} L^{I}
$$

with $\phi<1,0 \leq \theta<1, \tilde{\nu}:=\nu\left(L^{I}\right)^{-\theta}, \nu>0$, where $L^{I}$ is labor employed in innovative

\footnotetext{
${ }^{5}$ Employing the notation in section 2 , replace $x_{i}$ by $K_{i}^{Y}$ in (3) and use that, in equilibrium, $K_{i}^{Y}=K_{i}=x_{i}$.
} 
activities ("R\&D") and $\tilde{\nu}$ is taken as given by the representative $\mathrm{R} \& \mathrm{D}$ firm. In view of $(3)$, we have $G_{i}(\cdot)=Y^{i}$ and $\delta_{i}=\delta$ for accumulable factors $i \in\{1, \ldots, A\}$ and, from a social point of view, $G_{I}(\cdot)=\nu A^{\phi}\left(L^{I}\right)^{1-\theta}$ and $\delta_{I}=0$ in the process of knowledge accumulation (i.e. knowledge does not depreciate). ${ }^{6}$

The stock of knowledge $A$ enters as non-rival input into the knowledge accumulation process. Thus, parameter $\phi$ measures the extent of an intertemporal knowledge spillover (which is positive if $\phi>0$ ) and labor is the only rival R\&D input. An increase in $\theta$ means a larger wedge between the privately perceived constant-returns to R\&D and the socially declining marginal product of R\&D labor investment (higher "duplication externality").

Both the market for the final good and the labor market are perfect. Also the R\&D sector is perfectly competitive. Physical capital good producers possess market power but are restricted by a competitive fringe of firms which do not allow them to charge a mark-up higher than $\kappa \in(1,1 / \alpha] .^{7}$ In equilibrium, all capital good producers charge the same prices. Thus, $x_{i}=x$ and $\mathfrak{s}^{i}=Y^{i} / Y=\mathfrak{s}$ for all $i \in\{1, \ldots, A\}$. Let us define the total amount of physical capital as $K:=\sum_{i=1}^{A} x_{i}=A x$. Thus, aggregate output reads $Y=K^{\alpha}\left(A L^{Y}\right)^{1-\alpha}$. Moreover, summing (10) over all $i$ implies $\dot{K}=A \mathfrak{s} Y-\delta K$. Thus, the economy's aggregate investment rate in physical capital can be written as inv $:=(\dot{K}+\delta K) / Y=A \mathfrak{s}$. Initially, $x_{i}(0)=x_{0}>0$ for all $i \in\{1, \ldots, A\}$ and $A(0)=A_{0}>0$ are given.

Let $q=N c / Y, l^{Y}=L^{Y} / N$ and $l^{I}=L^{I} / N$. In the "reduced form", there are four allocation variables (i.e. $q$, inv, $l^{Y}$, and $l^{I}$ ) and two constraints on these allocation

\footnotetext{
${ }^{6}$ Moreover, $I^{A}=A$ and $I^{B}=1$, where capital is used only in final production.

${ }^{7}$ In addition to introducing investment subsidies, this is the only way we depart from Jones (1995) in this section. We introduce the competitive fringe in order to calibrate the mark-up factor according to empirical estimates.
} 
variables ${ }^{8}$ which may be stated as

$$
\begin{aligned}
& l^{Y}+l^{I}=1, \\
& q+i n v=1 .
\end{aligned}
$$

Hence, there are two degrees of freedom in the allocation variables.

The government may subsidize both $R \& D$ costs (R\&D sector) and capital costs ( $x$-sector). Subsidies are financed by a lump-sum tax levied on households. Profits are $\pi_{i}=\left[p_{i}-\left(1-s_{K}\right)(r+\delta)\right] x_{i}$ (capital producers) and $\Pi=P^{A} \tilde{\nu} A^{\phi} L^{I}-\left(1-s_{A}\right) w L^{I}$ (R\&D firms), where $p_{i}$ is the price of $x_{i}, r$ the interest rate, $P^{A}$ the price of blueprints for new physical capital goods, $w$ the wage rate, $s_{K}$ the subsidy on capital costs, and $s_{A}$ the subsidy on R\&D costs. Accounting for the competitive fringe, the mark up, charged by capital producers, is such that the equilibrium price is $p_{i}=\kappa\left(1-s_{K}\right)(r+\delta){ }^{9}$

There is mass one of infinitely-lived households with size $N$; initially, $N(0)=N_{0}>$ 0 . Household size grows at constant exponential rate $n \geq 0$. Preferences are represented by utility function (1). We employ the standard specification of instantaneous utility $u(c)=\frac{c^{1-\sigma}-1}{1-\sigma}$, with $\sigma>0$.

According to Proposition 1, the economy fulfills the necessary conditions for dynamic inefficiency. Suppose there indeed is underinvestment in $R \& D ;{ }^{10}$ that is, the socially optimal $R \& D$ subsidy rate is positive in the long run. The possibility to realize an intertemporal free lunch by increasing $\mathrm{R} \& \mathrm{D}$ subsidy rate, $s_{A}$, may exist for the following reason. On the one hand, an increase in the R\&D fraction of labor, $l^{I}$, lowers initial per capita income, $y(0):=Y(0) / N_{0}$. On the other hand, however, it could be the case that, at the same time, investment rate $\mathfrak{s}$ of intermediate goods producers declines as well. In this case, the resulting immediate decrease of inv may sufficiently

\footnotetext{
${ }^{8}$ In the unreduced form, allocation variables are $\left(c, x_{1}, \ldots, x_{A}, L^{Y}, L^{I}\right.$ \}, i.e., there are $V=A+3$ allocation variables and $R=A+1$ constraints (final goods market clearing condition and (10)). Again, $V-R=2$.

${ }^{9}$ See Grossmann, Steger and Trimborn (2010a).

${ }^{10}$ Even in the case where $\phi=\theta=0$, accumulation of $A$ is distorted downwards since innovators cannot fully appropriate the social gain from an innovation. That is, in equilibrium, the profit of an intermediate good firm, $\pi_{i}$, is smaller than the social return to an additional intermediate good, $\partial Y / \partial A$.
} 
raise the initial consumption rate $q(0)$ to induce an increase in initial consumption level $c(0)=q(0) y(0)$.

\subsection{Equilibrium}

To save space, we refer to Grossmann, Steger and Trimborn (2010a, Proposition 1) for the derivation of the equilibrium dynamics and the balanced growth equilibrium. ${ }^{11}$ Define $k:=K / N, p^{A}:=P^{A} / N$, and let $\tilde{z}:=z / N^{\frac{1-\theta}{1-\phi}}$ be the detrended value for any variable $z \in\{y, k, c, A\}$. It can be shown that the evolution of the market economy is governed by the following dynamic system (in addition to appropriate boundary conditions)

$$
\begin{aligned}
\tilde{A} & =\nu \tilde{A}^{\phi}\left(l^{I}\right)^{1-\theta}-g \tilde{A}, \\
\dot{p}^{A} & =(r-n) p^{A}-\frac{(\kappa-1)(\alpha / \kappa)^{\frac{1}{1-\alpha}}\left(1-l^{I}\right)}{\left[\left(1-s_{K}\right)(r+\delta)\right]^{\frac{\alpha}{1-\alpha}}}, \\
\dot{\tilde{k}} & =\left(\tilde{A}\left(1-l^{I}\right)\right)^{1-\alpha} \tilde{k}^{\alpha}-\tilde{c}-(\delta+n+g) \tilde{k}, \\
\dot{\tilde{c}} & =\frac{\tilde{c}(r-\rho)}{\sigma}-g \tilde{c} \\
r+\delta & =\frac{\alpha}{\kappa\left(1-s_{K}\right)}\left(\frac{\tilde{A}\left(1-l^{I}\right)}{\tilde{k}}\right)^{1-\alpha}, \\
p^{A} \nu \tilde{A}^{\phi-1}\left(l^{I}\right)^{-\theta} & =\left(1-s_{A}\right)(1-\alpha)\left(\frac{\tilde{k}}{\tilde{A}\left(1-l^{I}\right)}\right)^{\alpha} .
\end{aligned}
$$

The steady state may be characterized by $\dot{k} / k=\dot{c} / c=\dot{A} / A=\frac{(1-\theta) n}{1-\phi}=: g$ and $\dot{l}^{I} / l^{I}=\dot{p}^{A} / p^{A}=0$.

We now analyze the model numerically by employing the analytically derived balanced growth equilibrium and the differential equation system. The numerical simulations rest on calibration of the model to the US. Transitional dynamics are calculated numerically by applying the relaxation algorithm (Trimborn, Koch and Steger, 2008).

\footnotetext{
${ }^{11}$ Grossmann et al. (2010a) derive the dynamically optimal policy mix in the model presented in this section. They do not analyze the possibility of dynamic inefficiency, however.
} 


\subsection{Calibration}

The observable parameters/ variables largely match the characteristics of the US economy under the assumption that the US is in steady state. ${ }^{12}$ We take a per capita long run output growth rate $(g)$ of 2 percent, a population growth rate $(n)$ of 1 percent, and a long run interest rate $(r)$ of 7 percent. Given the time preference $\rho$, parameter $\sigma$ is determined by the Keynes-Ramsey rule $\dot{c} / c=g=(r-\rho) / \sigma$. The depreciation rate $\delta$ can be inferred from US investment rate $(i n v)$ and the capital-output ratio $(K / Y)$. We use that inv $=(\dot{K}+\delta K) / Y=(\dot{K} / K+\delta) K / Y$. In steady state, $\dot{K} / K=n+g$; thus, $i n v=(n+g+\delta) K / Y$. For the US, we observe $i n v=0.21$ and $K / Y=3$, leading to $\delta=0.04$. The output elasticity of capital $(\alpha)$ is given by the condition that the user cost of capital $(r+\delta)$ equals the marginal product of capital under optimal capital investment: $\alpha=(r+\delta) K / Y$. In fact, we assume that the capital subsidy rate ensures optimal capital investment at all times, $s_{K}=1-1 / \kappa$ (Grossmann et al., 2010a, Proposition 2). The mark up factor $\kappa$ is set to an intermediate value of the empirically plausible range $\kappa \in[1.05,1.4]$ (Norrbin, 1993): $\kappa=1.2$. A higher mark up factor mitigates the well known "surplus appropriability problem", which gives rise to $R \& D$ underinvestment, but aggravates underinvestment in physical capital. The $\mathrm{R} \& \mathrm{D}$ underinvestment problem is enhanced the higher is $\phi$ (intertemporal spillover) and mitigated the higher is $\theta$ (duplication externality). If $\theta$ is close to one, there may be R\&D overinvestment. We assume an intermediate value $\theta=0.5$. Both $\phi$ and $\theta$ are not independent from each other and set to match the economy's steady state growth rate, $g=\frac{(1-\theta) n}{1-\phi}$. The current US R\&D subsidy rate is just slightly above zero $\left(s_{A}=0.066\right)$. Table 1 below summarizes our calibration.

As a result of the calibration, the implied R\&D intensity is as observed for business $\mathrm{R} \& \mathrm{D}$ in the US: $w L^{I} / Y \simeq 0.02$. The optimal $\mathrm{R} \& \mathrm{D}$ subsidy rate which implements the optimal long run labor allocation is fairly high: $s_{A}=0.88$. It reflects severe R\&D underinvestment. The sectoral misallocation of labor is the underlying source for the possibility of an intertemporal free lunch. The optimal rate $s_{A}=0.88$ implies a (first

\footnotetext{
${ }^{12}$ The calibration strategy largely follows Grossmann et al. (2010a).
} 
best) $R \& D$ intensity of about 0.15 in the long run. ${ }^{13}$

\begin{tabular}{llllll}
\hline Parameter & Value & Source & Parameter & Value & Source \\
\hline \hline$g$ & 0.02 & PWT 6.2 (Heston et al., 2006) & $\sigma$ & 2.5 & implied \\
$n$ & 0.01 & PWT 6.2 (Heston et al., 2006) & $\kappa$ & 1.2 & Norrbin (1993) \\
$\delta$ & 0.04 & implied & $s_{A}$ & 0.066 & OECD (2009) \\
$r$ & 0.07 & Mehra and Prescott (1985) & $s_{K}$ & $1 / 6$ & first best value \\
$\alpha$ & 0.33 & implied & $\theta$ & 0.5 & intermediate value \\
$\rho$ & 0.02 & "usual value" & $\phi$ & 0.75 & implied \\
\hline
\end{tabular}

Table 1: Calibration

\subsection{The Intertemporal Free Lunch}

It is assumed that the economy is in a steady state initially with $s_{A}=0.066$. Figure 1 shows the time path of detrended and normalized per capita consumption in response to an increase to $s_{A}=0.6 .^{14}$ It illustrates that initial consumption jumps upwards by about 3 percent instantaneously and is never lower than the initial steady state value (normalized to unity). Per capita consumption increases drastically by about 370 percent in the long run.

The economic intuition for the possibility of an intertemporal free lunch is as follows. The increase in $s_{A}$ causes the fraction of labor allocated to $\mathrm{R} \& \mathrm{D}, l^{I}=1-l^{Y}$, to increase. Due to the decline in the fraction of labor in manufacturing $\left(l^{Y}\right)$, per capita output $y$ drops initially (labor reallocation effect). However, the expansionary R\&D policy attenuates the substantial R\&D underinvestment in the market economy. Rational,

\footnotetext{
${ }^{13}$ The numerically derived optimal policy mix is quite robust to extensions of the model. Grossmann, Steger and Trimborn (2010b) derive similar values for the behaviorally relevant subsidy rates when accounting for (i) endogenous human capital accumulation, (ii) distortionary income taxation, (iii) business stealing effects from R\&D (following Jones and Williams, 2000), and (iv) transitional dynamics. Moreover, Grossmann and Steger (2011) show that allowing for heterogeneity of R\&D skills leaves the analytical solution for the optimal long run subsidy mix unaffected.

${ }^{14}$ The employed numerical method by Trimborn et al. (2008), which underlies Fig. 1, does not rely on linearization around the steady state, which would be inappropriate for the question at hand. The algorithm is implemented in Mathematica. The underlying file is available from the authors upon request.
} 
forward-looking agents understand that there is an associated wealth effect. They therefore reduce the fraction of output devoted to the accumulation of physical capital, i.e. inv decreases. Consequently, the rate of consumption, $q=1-i n v$, rises. If the increase in $q(0)$ is large relative to the decrease in initial per capita income, $y(0)$, per capita consumption $c(0)=q(0) y(0)$ may jump up initially.
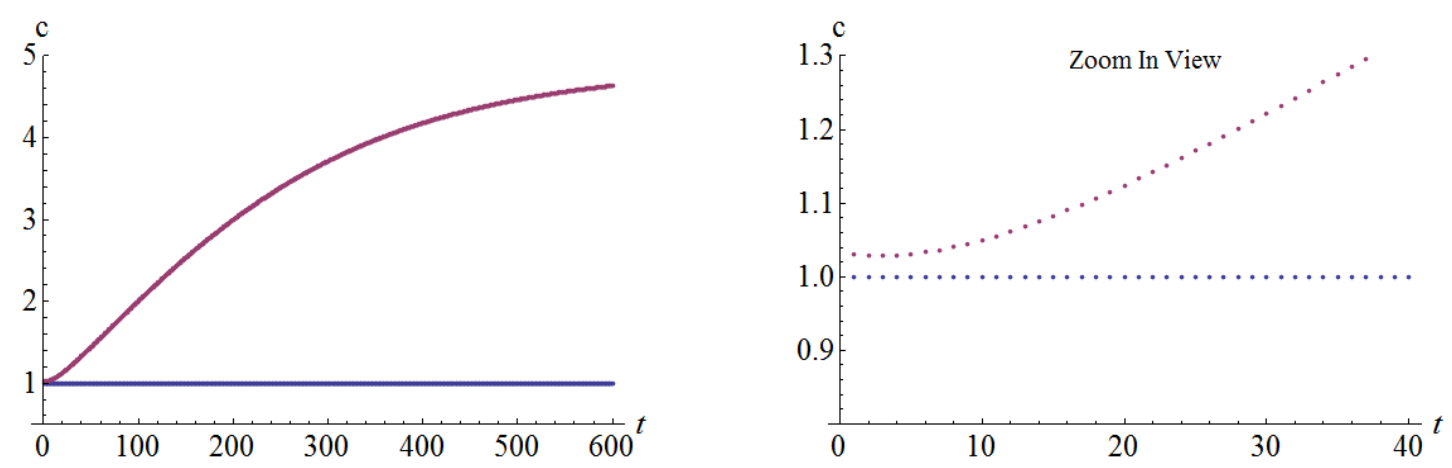

Figure 1: The intertemporal free lunch

Notice that the Jones (1995) model fulfills the conditions of Proposition 1: (i) raising the R\&D subsidy affects the accumulation of knowledge, (ii) there are two degrees of freedom in the allocation variables. Also the presumption of Proposition 1 holds, as the optimal long run R\&D subsidy is positive.

How does the proportional initial change of consumption (the 3 percent in the example considered above) depend on the policy instrument $s_{A}$ ? To see this, consider the initial (i.e. at $t=0$ ) rate of change of detrended per capita consumption, $\Delta \tilde{c}(0) / \tilde{c}(0)$, in response to a change in $s_{A}$ from $s_{A}=0.066$ to $s_{A} \in[0.066-0.3,0.066+0.85]$. By construction, at initial (US) value $s_{A}=0.066$, we have $\Delta \tilde{c}(0)=0$. Figure 2 (a) shows $\Delta \tilde{c}(0) / \tilde{c}(0)$ as function of the $\mathrm{R} \& \mathrm{D}$ subsidy. It shows that $\Delta \tilde{c}(0) / \tilde{c}(0)$ is rising in $s_{A}$ up to $s_{A}=0.72$ and is negative for $s_{A}$-increases slightly beyond the socially optimally rate, i.e. for $s_{A}>0.89 .{ }^{15}$

\footnotetext{
${ }^{15}$ The range of $s_{A}$-increases which generate an increase in $\tilde{c}(0)$ is smaller when the initial $\mathrm{R} \& \mathrm{D}$ underinvestment problem is less severe. For instance, if the "duplication externality" is parameterized by $\theta=0.75$, the steady state $R \& D$ intensity at the socially optimal $R \& D$ subsidy rate is 7.9 percent (rather than 15 percent in the case where $\theta=0.5$ ). In this case, $\tilde{c}(0)$ becomes negative for increases in $s_{A}$ to a level which is considerably smaller than the socially optimal rate (not shown). However, qualitatively, the insights from Figure 2 remain valid.
} 

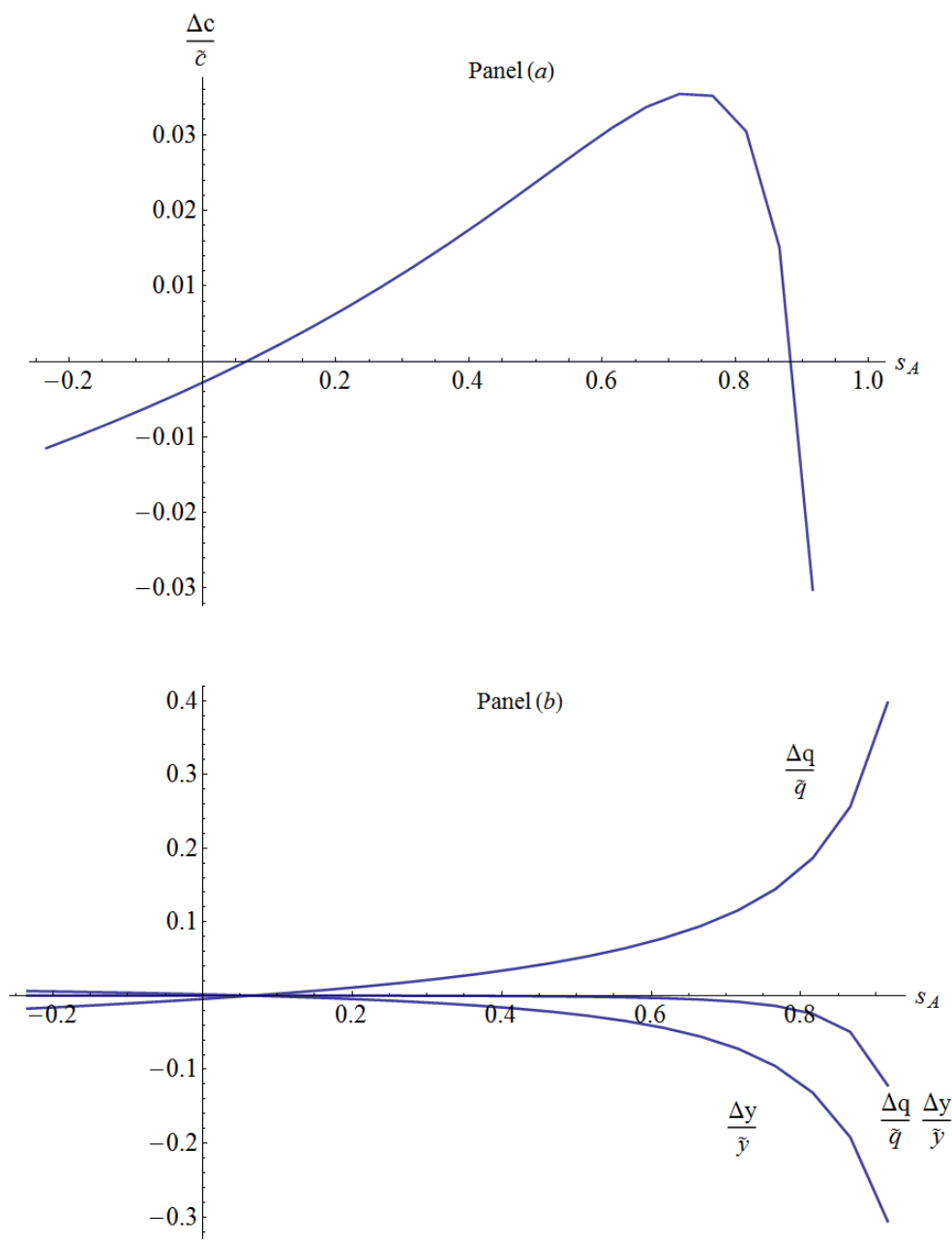

Figure 2: The instantaneous impact of an increase in the R\&D subsidy

To further clarify the mechanics of an intertemporal free lunch, let us decompose the rate of change of detrended per capita consumption by using the definition of the consumption rate, $q=c / y=\tilde{c} / \tilde{y}$. Using $\tilde{c}=q \tilde{y}$ we have

$$
\frac{\Delta \tilde{c}(0)}{\tilde{c}(0)}=\frac{\Delta q(0)}{q(0)}+\frac{\Delta \tilde{y}(0)}{\tilde{y}(0)}+\frac{\Delta q(0)}{q(0)} \cdot \frac{\Delta \tilde{y}(0)}{\tilde{y}(0)} .
$$

Figure 2 (b) shows the three terms of the right-hand side as function of $s_{A}$. We confirm that, for $s_{A}>0.066$, the proportional change in the consumption rate $q(0)$ is positive and increasing in $s_{A}$, whereas the proportional change in (detrended) per capita output $y(0)$ is negative and decreasing in $s_{A}$. When $s_{A}$ is not too high, the rise in the consumption rate is rather large relative to the drop in per capita output, 
implying $\Delta \tilde{c}(0)>0$.

\section{Dynamic Inefficiency and Endogenous Growth}

This section briefly discusses whether alternative, widely-used endogenous growth models satisfy the necessary conditions for dynamic inefficiency, as listed in Proposition 1.

\subsection{Lab-equipment Approach}

Romer and Rivera-Batiz (1991) propose a R\&D-based growth model which differs from the set up analyzed in the previous section with respect to the R\&D technology. Horizontal innovations occur according to

$$
\dot{A}=\eta Y^{I}-\delta_{I} A,
$$

where $Y^{I}$ is the amount of final output used for knowledge accumulation. Capturing the R\&D process in this way is sometimes labelled as "lab-equipment approach". ${ }^{16}$ Labor is used for producing final output only, i.e., in equilibrium $l^{Y}=1$. We can now distinguish between three income shares to represent the use of income:

$$
q+i n v+\mathfrak{s}^{I}=1
$$

The model fulfills conditions (i) and (ii) in Proposition 1 such that dynamic inefficiency may occur. For instance, an increase in the capital cost subsidy raises the aggregate investment rate into physical capital, inv. If, at the same time, the R\&D investment rate, $\mathfrak{s}^{I}=Y^{I} / Y$, declines due to a wealth effect similar to the one discussed above, then the net effect on the initial consumption rate, $q(0)$, may be positive. If it is, per capita consumption $c(0)$ would increase since per capita output is not affected instantaneously after a policy intervention. Thus, an intertemporal free lunch may be

\footnotetext{
${ }^{16}$ According to the classification in section 2, in contrast to the Jones (1995) model, the approach constitutes a one-sector model. Only final output is used in the accumulation processes.
} 
possible.

\subsection{Learning-by-doing}

We next consider learning-by-doing externalities à la Arrow (1962) and Romer (1986). For illustrative purposes, consider the following production function of a representative final goods producer:

$$
Y=A a\left(K^{Y}\right)^{\alpha}\left(L^{Y}\right)^{1-\alpha}
$$

$0<\alpha<1, A>0$, where productivity measure $a:=\left(K^{Y}\right)^{\beta}, \beta>0$, is taken as given by the $Y$-sector and driven by the aggregate capital input, $K^{Y}$. The assumption captures that final goods producers do not take into account that capital investment raises the economy-wide capital stock and therefore enhances total factor productivity. This "learning-by-doing" externality distorts accumulation of the only capital good downwards. With endogenous investment rate $\mathfrak{s}$, capital accumulates according to $\dot{K}=\mathfrak{s} Y-\delta K, \delta>0$. There is no other sector such that, in equilibrium, $K^{Y}=K$ and $L^{Y}=N$ (full employment conditions). For instance, if total labor supply $N=1$ and $\beta=1-\alpha$, the social production function is $Y=A K$ ("AK-model").

The consumption rate reads $q=1-\mathfrak{s}$. Now, addressing the externality by a policy intervention which raises $\mathfrak{s}$ leaves per capita output initially unaffected but inevitably lowers $q(0)$. Thus, an intertemporal free lunch is not possible and, by design, the economy is dynamically efficient. Relating the insight to Proposition 1, note that there is only one degree of freedom in the allocation variables. That is, $V=4$ and $R=3$ (full employment conditions and final goods market clearing). Thus, condition (ii) of Proposition 1 is violated.

Whether the model is interpreted to encompass one or two accumulable input factors would not change the conclusion. One may consider TFP, as given by $a:=K^{\beta}$ (let $A=1$ ), as the second accumulable factor such that $\dot{a}=\beta K^{\beta-1} \dot{K}$. However, this model version still violates condition (ii) of Proposition 1. It illustrates that the existence of two degrees of freedom is indeed necessary for an intertemporal free lunch to be realized, even in the case with two accumulable factors $(I=2)$. 


\subsection{Human Capital Externalities}

Finally, consider human capital externalities à la Uzawa (1965) and Lucas (1988). Let the production technology for final output be a function of both physical capital $(K)$ and human capital $(H)$ such that $Y=b\left(K^{Y}\right)^{\alpha}\left(H^{Y}\right)^{1-\alpha}, 0<\alpha<1$. The per capita amount of human capital in the economy is denoted by $h$. There is a human capital externality which drives total factor productivity, $b:=h^{\gamma}, \gamma>0$. Unlike in the previous examples, now the accumulation process of human capital uses neither final output nor a non-accumulable factor but human capital itself. The education technology reads

$$
\dot{h}=\xi\left(h^{H}\right)^{\eta}-\delta_{H} h
$$

$\xi>0, \eta>0, \delta_{H} \geq 0$, where $h^{H}$ is the per capita level of human capital devoted to education. ${ }^{17}$ The associated resource constraint reads

$$
h^{H}+h^{Y}=h,
$$

where $h^{Y}$ is the per capita level of human capital devoted to final goods production. Physical capital accumulation is standard in its exclusive use of final output; we have $\dot{K}=\mathfrak{s} Y-\delta_{K} K, \delta_{K} \geq 0$. Thus, the consumption rate again reads $q=1-\mathfrak{s}$.

The Lucas-Uzawa model fulfills the necessary conditions for an intertemporal free lunch mentioned in Proposition 1. There are two accumulable factors, namely $K$ and $h$. Moreover, there are five allocation variables $\left(K^{Y}, \mathfrak{s}, q, h^{H}, h^{Y}\right)$ and three constraints, namely $K^{Y}=K, \mathfrak{s}+q=1$ and (25), such that there are two degrees of freedom in the allocation variables. Due to the human capital externality $(\gamma>0)$, an education subsidy, which raises $h^{H}$, may be justified. However, this inevitably reduces initial per capita output $y(0)$, in view of constraint (25). Nonetheless, it may be that, at the same time, the education subsidy induces a wealth effect which leads to a decline in investment rate $\mathfrak{s}$. Consequently, an instantaneous increase in the consumption

\footnotetext{
${ }^{17}$ Lucas (1988) implicitly assumes constant returns in the education technology, $\eta=1$, which allows for sustained endogenous growth.
} 
rate, $q(0)$, is possible and may be high enough to raise the initial level of per capita consumption, $c(0)=q(0) y(0)$.

\section{Conclusion}

This paper has identified necessary conditions of dynamic inefficiency in the widelyused class of dynamic, general equilibrium, infinite-horizon models with optimizing agents even in a situation with long run underinvestment in an accumulable factor. To the best of our knowledge, the possibility to realize an intertemporal free lunch in such a framework has not been discussed in the previous literature. Our paper aims to fill this gap. In order to dynamically evaluate policy interventions, researchers have to be aware whether they are employing a framework where the possibility of dynamic inefficiency is built-in or not.

We illustrated the possibility of an intertemporal free lunch in a standard, general equilibrium model à la Jones (1995) with R\&D-based, semi-endogenous, long run growth and accumulation of physical capital. Interestingly, although the long run rate of economic growth is not policy-dependent in the analyzed framework, we find that the US economy underinvests in R\&D and is dynamically inefficient. That is, TANSTAAFL does not apply. At the first glance, this seems surprising. A priori, one would suspect that mitigating the $R \& D$ underinvestment problem requires to give up consumption at least in the short run. We showed that this is not the case when the $R \& D$ subsidy rate is raised, due to an immediate slowdown in the process of capital accumulation.

In sum, our analysis suggests that dynamic inefficiency is not a theoretical anomaly even in models with fully optimizing, infinitely-lived, forward-looking agents and underinvestment. It is not often that economists can make such strong policy recommendations than in the case of dynamic inefficiency. Thus, future work should further investigate this fascinating issue in order to help realizing possible intertemporal free lunches. 


\section{References}

[1] Abel, Andrew, Gregory M. Mankiw, Lawrence H. Summers and Richard J. Zeckhauser (1989). Assessing Dynamic Efficiency: Theory and Evidence, Review of Economic Studies 56, 1-19.

[2] Arrow, Kenneth J. (1962). The Economic Implications of Learning By Doing, Review of Economic Studies 29, 155-173.

[3] Friedman, Milton (1975). There's No Such Thing as a Free Lunch, LaSalle, Ill.: Open Court.

[4] Grossmann, Volker, Thomas M. Steger and Timo Trimborn (2010a). Dynamically Optimal R\&D Subsidization, CESifo Working Paper No. 3153.

[5] Grossmann, Volker, Thomas M. Steger and Timo Trimborn (2010b). Quantifying Optimal Growth Policy, CESifo Working Paper No. 3092.

[6] Grossmann, Volker and Thomas M. Steger (2011). Optimal Policy Towards Growth and Innovation: The Role of Skill Heterogeneity, University of Fribourg and Leipzig (mimeo).

[7] Heston, Alan, Robert Summers and Bettina Aten (2006). Penn World Table Version 6.2, Center for International Comparisons of Production, Income and Prices at the University of Pennsylvania.

[8] Jones, Charles I. (1995). R\&D-based Models of Economic Growth, Journal of Political Economy 103, 759-784.

[9] Jones, Charles I. and John C. Williams (2000). Too Much of a Good Thing? The Economics of Investment in R\&D, Journal of Economic Growth 5, 65-85.

[10] Lucas, Robert E. (1988). On the Mechanics of Economic Development, Journal of Monetary Economics 22, 3-42. 
[11] Mankiw, N. Gregory, David Romer and David N. Weil (1992). A Contribution to the Empirics of Economic Growth, Quarterly Journal of Economics 107, 407-437.

[12] Mehra, Rajnish and Edward C. Prescott (1985). The Equity Premium: A Puzzle, Journal of Monetary Economics 15, 145-61.

[13] Norrbin, Stefan C. (1993). The Relationship Between Price and Marginal Cost in U.S. Industry: A Contradiction, Journal of Political Economy 101, 1149-1164.

[14] OECD (2009). Science Technology and Industry Scoreboard 2009, Paris.

[15] Phelps, Edmund S. (1966). Golden Rules of Economic Growth, New York: W.W. Norton.

[16] Romer, Paul M. (1986). Increasing Returns and Long-run Growth, Journal of Political Economy 94, 1002-1037.

[17] Romer, Paul M. and Luis A. Rivera-Batiz (1991). Economic Integration and Endogenous Growth, Quarterly Journal of Economics 106, 531-555.

[18] Trimborn, Timo, Karl-Josef Koch and Thomas M. Steger (2008). MultiDimensional Transitional Dynamics: A Simple Numerical Procedure, Macroeconomic Dynamics 12, 301-319. 